original surgery. ${ }^{5}$ Our patient presented with a graft-enteric fistula almost 2 years after the repair of his aneurysm. This may have resulted from mechanical erosion by the graft through the bowel wall, but is more likely to have followed a primary graft infection. We think that the graft in our patient became infected during a transient bacteraemia, the original source of which is uncertain.

Since the 8-week course of ciprofloxacin in 1990, there have been no further episodes of arthritis and there was no evidence of graft infection when he was assessed in 1992. Ciprofloxacin penetrates well into tissues and has high bioavailability, enabling it to be used as oral therapy for Pseudomonas aeru- ginosa infections. ${ }^{6}$ The antibiotic therapy in 1988 is unlikely to have eradicated Pseudomonas aeruginosa from his graft but the latest prolonged course with ciprofloxacin appears to have successfully cleared the organism.

Previous reports of septic arthritis caused by Gram-negative bacilli emphasized the poor outcome associated with this condition. ${ }^{7}$ A more recent study found that the prognosis has improved considerably even without surgical intervention. ${ }^{2}$ This case demonstrates that joint aspiration combined with antibiotic therapy can achieve a good outcome in Gram-negative septic arthritis with restoration of normal joint function.

\title{
References
}

1. Cooper, C. \& Cawley, M.I.D. Bacterial arthritis in an English health district: a 10 year review. Ann Rheum Dis 1986, 45: 458-463.

2. Newman, E.D., Davis, D.E. \& Harrington, T.M. Septic arthritis due to gram negative bacilli: older patients with good outcome. J Rheumatol 1988, 15: 659-662.

3. Miskew, D.B.W., Lorenz, M.A., Pearson, R.L. \& Pankovich, A.M. Pseudomonas aeruginosa bone and joint infections in drug abusers. J Bone Joint Surg 1983, 65A: 829-832.

4. Chusid, M.J., Jacobs, W.M. \& Sty, J.R. Pseudomonas arthritis following puncture wounds of the foot. J Pediatr 1979, 94: 429-431.

5. O'Brien, T. \& Collin, J. Prosthetic vascular graft infection. Br J Surg 1992, 79: 1262-1267.

6. Hooper, D.C. \& Wolfson, J.S. Fluoroquinolone antimicrobial agents. $N$ Engl J Med 1991, 324: 384-394.

7. Goldenberg, D.L., Brandt, K.D., Cathcart, E.S. \& Cohen, A.S. Acute arthritis caused by Gram-negative bacilli: a clinical characterization. Medicine 1974, 53: 197-208.

\section{Multilobated B-cell lymphoma of the spleen}

\author{
D.J. Farrell, C.A. Bloxham and H.H. Lucraft ${ }^{1}$ \\ Department of Histopathology and ${ }^{1}$ Department of Clinical Oncology, Newcastle General Hospital, \\ Newcastle upon Tyne, UK
}

\begin{abstract}
Summary: We report a case of multilobated B-cell lymphoma presenting with primary splenic involvement. This is a very unusual tumour occurring in an uncommon site.
\end{abstract}

\section{Introduction}

Peripheral T-cell lymphomas characterized by cells having large multilobated nuclei were first des-

Correspondence: D.J. Farrell, B.A., M.B., B.Ch., Freeman Hospital, High Heaton, Newcastle-upon-Tyne NE7 7DH, UK.

Accepted: 17 May 1993 cribed by Pinkus et al. in 1979. ${ }^{1}$ Subsequently multilobated non-Hodgkin's lymphomas with a B-cell phenotype were reported. ${ }^{2,3}$ These tumours may occur in nodal, extranodal or combined locations but most frequently present in an extranodal site. We report a case presenting with hypogammaglobulinaemia amd splenomegaly. This is a very rare occurrence. 


\section{Case history}

A 37 year old man was found to have hypogammaglobulinaemia in 1989 following investigation for recurrent chest infections. He began treatment with weekly immunoglobulin injections. In July 1990 he developed severe pain in his left posterior lower chest and drenching night sweats. He was found to have splenomegaly. Computed tomographic (CT) scan confirmed that the spleen was enlarged and contained multiple soft tissue non-enhancing nodules and also showed multiple enlarged lymph nodes in the mesentery and around the origin of the superior mesenteric artery. The liver appeared radiologically normal. CT scan of the chest showed no evidence of disease and there was no palpable lymphadenopathy. Diagnostic laparotomy and splenectomy was performed in November 1990. Preoperative blood count was normal. Bone marrow aspirate and trephine showed reactive hypocellular marrow with no evidence of lymphoma. Lactate dehydrogenase was raised at $759 \mathrm{U} / 1$ (normal less than $430 \mathrm{U} / 1$ ).

A clinical diagnosis of Stage IIB high-grade non-Hodgkin's lymphoma was made and he was treated with combination chemotherapy (cyclophosphamide, vincristine, adriamycin and prednisolone in pulsed courses). He was in complete remission at completion of chemotherapy in March 1991. He has subsequently remained well with no evidence of further disease (to February 1993). Hypogammaglobulinaemia has persisted and he continues to receive gammaglobulin replacement.

\section{Pathological findings}

Macroscopic examination showed a spleen weighing $530 \mathrm{~g}$ and measuring $16 \times 11 \times 5 \mathrm{~cm}$. The surface of the spleen was nodular. On sectioning there were many white tumour nodules which were irregular in outline but well demarcated and measuring up to $2.5 \mathrm{~cm}$ in maximum dimension. Within the splenic hilum and the attached omentum there were several small lymph nodes measuring up to $1 \mathrm{~cm}$ in diameter which had a grey cut surface. Microscopic examination showed that the tumour deposits were composed of large pleomorphic lymphoid cells with vesicular nuclei, prominent large round eosinophilic nucleoli and variable amounts of eosinophilic cytoplasm (Figure 1), which comprised up to $53 \%$ of the malignant infiltrate. The nuclei of many tumour cells were markedly irregular with deep clefts and an occasional clover leaf pattern (Figure 2) characteristic of the multilobated lymphoma. Scattered multinucleated cells were also present but classical Reed-Sternberg cells were not identified. Foci of necrosis and fibrosis were present throughout and

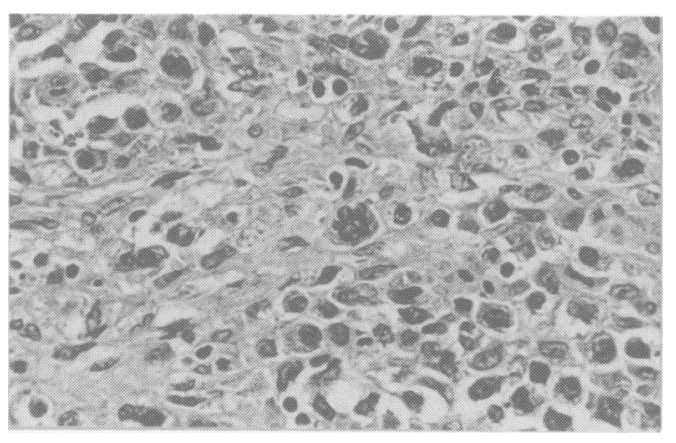

Figure 1 The tumour is composed predominantly of lymphoid cells with large pleomorphic nuclei. Original magnification, $\times 400$. Haematoxylin and eosin.

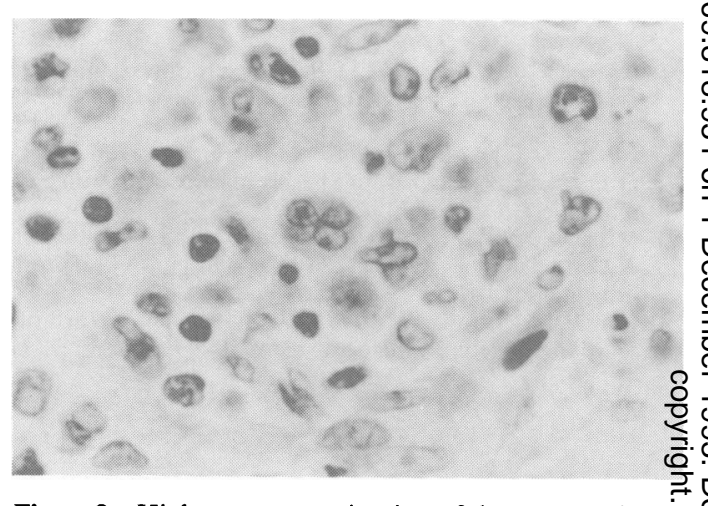

Figure 2 High-power examination of the tumour shows that some cells have a multilobated appearance with deep clefts separating the lobes. Original magnification, $\times 1000$. Haematoxylin and eosin.

in areas only a perivascular ring of viable tumour cells was seen. A T-cell lymphoma was thought most likely on morphological grounds but immunohistochemistry of the tumour cells showed positive staining using the monoclonal antibodies MB1 (CD45RA), MB2 and L26 (CD20), and negative staining for MT1 (CD43) and UCHL1 (CD45RO). Staining for CD30 and CD15 was also negative. Thus the immunohistochemical profile was consistent with B-cell phenotype lymphoma The lymph nodes recovered from the splenic hilum and omentum were not involved by the tumour which suggests that the malignancy may have been confined to the spleen. However, the enlarged mesenteric lymph nodes noted on CT scan were not biopsied.

\section{Discussion}

Multilobated non-Hodgkin's lymphomas are a well recognized though uncommon entity. The 
original case reports suggested that these were T-cell lymphomas ${ }^{1}$ but subsequent phenotyping has shown that both $\mathrm{B}$-cell and $\mathrm{T}$-cell varieties exist. $^{2,3}$

Several series of multilobated B-cell lymphoma have been reported ${ }^{3-5}$ and these tumours, similar to their T-cell counterparts, present preferentially as extranodal lesions. However, splenomegaly is a very rare presenting feature. Van Baarlen et al. ${ }^{3}$ and O'Hara et al. $^{4}$ presented 30 and nine cases, respectively, in which this tumour involved a wide variety of tissues such as parotid, maxilla, soft tissues, bone and testis among others, but none of these cases showed splenic involvement. Indeed, to our knowledge there has only been one previous case report of multilobated B-cell lymphoma presenting with primary splenic involvement, ${ }^{2}$ making this case a very unusual site of presentation for this unusual tumour. It is important to recognize this rare morphological subtype, particularly when arising in an extranodal site, as prognosis for multilobated lymphomas appears to be rather favourable with a striking incidence of long-term remission even after inadequate local therapy. ${ }^{3}$

Hypogammaglobulinaemia is seen to a greater or lesser extent in all B-cell neoplasms. Therefore, if hypogammaglobulinaemia is discovered in any patient above the age of 20 which cannot be explained through other causes, it should be taken to indicate B-cell neoplasia until proved otherwise. ${ }^{6}$

\section{References}

1. Pinkus, G.S., Said, J.W. \& Hargreaves, H. Malignant lymphoma, T-cell type. A distinct morphologic variant with multilobated nuclei, with a report of four cases. Am J Clin Pathol 1979, 72: 540-550.

2. Palutke, M., Schnitzer, B., Mirchandani, I. et al. T- and B-cell lymphomas look alike (letter to the editor). Am J Clin Pathol 1980, 74: 360-361.

3. Van Baarlen, J., Schuurman, J.H. \& Van Unnik, J.A. Multilobated non-Hodgkin's lymphoma. A clinicopathologic entity. Cancer 1988, 61: 1371-1376.

4. O'Hara, C.J., Said, J.W. \& Pinkus, G.S. Non-Hodgkin's lymphoma, multilobated B-cell type: report of nine cases with immunohistochemical and immunoultrastructural evidence for a follicular centre cell derivation. Hum Pathol 1986, 17: 593-599.
It is suggested that in B-cell neoplasms physiological regulators of antibody responses (possibly suppressor T-cells) are activated, which selectively moderate B-cell triggering by antigen. Other suggested possible mechanisms are increased catabolism of immunoglobulins ${ }^{7}$ and induced hypersplenism with consequent antigen capture and breakdown. ${ }^{8}$ The reduced immunoglobulin levels impair primary antibody responses more profoundly than secondary responses and patients are susceptible to pyogenic infections, especially chest infections, as occurred in this case.

In summary, multilobated lymphomas are a distinct morphological subtype which may be of B-cell or T-cell origin. They often present in extranodal locations and have a good prognosis. The phenotype of these large cell lymphomas cannot be determined with certainty by the morphological appearances of the tumour cells and immunocytochemistry is required for a definite diagnosis of phenotype. This case is unusual in that this is an uncommon type of lymphoma presenting in an unusual manner and which appears to be confined to the spleen.

\section{Acknowledgement}

We wish to thank Dr B. Angus for his comments, Mr I. Taylor for producing the photographs and Mrs E. Tweedy for typing the manuscript.

5. Chan, J.K., Ng, C.S. \& Tung, S. Multilobated B-cell lymphoma, a variant of centroblastic lymphoma. Report of four cases. Histopathology 1986, 10: 601-612.

6. Galton, D.A. \& MacLennan, I.C. Clinical patterns in B lymphoid malignancy. Clin Haematol 1982, 11: 561-587.

7. Lippincott, S.H., Korman, S., Ford, L., Stickley, E., Wolins, W. \& Hughes, W.L. Turnover of labelled normal gammaglobulin in multiple myeloma. J Clin Invest 1960, 39: 565-572.

8. Joshua, D.E., Brown, G. \& MacLennan, I.C. Immune suppression in Balb/c mice bearing the plasmacytoma TEPC183: evidence for normal lymphocytes but defective macrophage function. Int J Cancer 1979, 231: 663-672. 